\title{
ON THE STEPANOV ALMOST PERIODIC SOLUTION OF A SECOND-ORDER INFINITESIMAL GENERATOR DIFFERENTIAL EQUATION
}

\author{
ARIBINDI SATYANARAYAN RAO \\ Department of Mathematics, Concordia University \\ Montreal, P. Quebec, Canada, H3G 1M8 \\ (Received April 5, 1990 and in revised form January 14, 1991)
}

\begin{abstract}
The Stepanov almost periodic solution of a certain second-order differential equation in a reflexive Banach space is shown to be almost periodic.
\end{abstract}

KEY WORDS AND PHRASES: Bochner (Stepanov) almost periodic function, bounded linear operator, strongly continuous group, infinitesimal generator.

1980 AMS SUBJECT CLASSIFICATION CODE. PRIMARY 34GXX，34G10，34C27; SECONDARY $47 \mathrm{D} 10$.

1. INTRODUCTION.

Let $X$ be a Banach space and $J$ the interval $-\infty<t<\infty$. A continuous function $f: J \rightarrow X$ is said to be (Bochner or strongly) almost periodic if, given $\epsilon>0$, there exists a positive real number $r=r(\epsilon)$ such that any interval of the real line of length $r$ contains at least one point $\tau$ for which

$$
\sup _{t \in J}|f(t+\tau)-f(t)| \leq \epsilon .
$$

A function $f \in L_{\text {loc }}^{p}(J ; X)$ with $l \leq p<\infty$ is said to be stepanovbounded or $S^{P_{-}}$bounded on $\mathrm{J}$ if

$$
\|f\|_{S^{p}}=\sup _{t \in J}\left[\int_{t}^{t+1} \mid f(s) \mathbb{P}^{p} d s\right]^{1 / p}<\infty .
$$

A function $f \in L_{10 c}^{p}(J ; X)$ with $1 \leq p<\infty$ is said to be stepanov almost periodic or $S^{P_{-}}$almost periodic if, given $\epsilon>0$, there is a positive real number $r=r(\epsilon)$ such that any interval of the real line of length $r$ contains at least one point $\tau$ for which

$$
\sup _{t \in J}\left[\int_{t}^{t+1}|f(s+\tau)-f(s)|^{p} d s\right]^{1 / p}<\infty .
$$

We denote by $L(X, X)$ the set of all bounded linear operators on $X$ into itself, with the uniform operator topology. An operator-valued function $T: J \rightarrow L(X, X)$ is called a strongly continuous group if 


$$
\begin{aligned}
& T\left(t_{1}+t_{2}\right)=T\left(t_{1}\right) T\left(t_{2}\right) \text { for all } t_{1}, t_{2} \in J ; \\
& T(0)=I=\text { the identity operator on } X ;
\end{aligned}
$$

for each $x \in X, T(t) x, t \in J \rightarrow X$ is continuous.

The infinitesimal generator $A$ of a strongly continuous group $T: J \rightarrow L(X, X)$ is a closed linear operator, with domain $D(A)$ dense in $X$, defined by

$$
A x=\lim _{t \rightarrow 0} \frac{T(t) x-x}{t} \text { for } x \in D(A)
$$

(see Dunford and Schwartz [3]).

The group $T$ is said to be almost periodic if $T(t) x, t \in J \rightarrow X$ is almost periodic for each $x \in X$.

NOTE 1. Suppose $A$ and $B$ are two densely-defined closed linear operators, having their domains and ranges in a Banach space $X$, and a function $f: J \rightarrow X$ is continuous. Then a solution of the differential equation

$$
u^{\prime \prime}(t)=A u^{\prime}(t)+B u(t)+f(t) \quad \text { a.e. on } \mathrm{J}
$$

is a twice differentiable function $u(t)$ with $u^{\prime}(t) \in D(A), u(t) \in D(B)$ for all $t \in J$ and satisfying the equation (1.8) a.e. (almost everywhere) on $\mathrm{J}$.

our result is as follows.

THEOREM. Suppose $X$ is a reflexive Banach space, $f: J \rightarrow X$ is an $S^{1}$ - almost periodic continuous function, and $A$ is the infinitesimal generator of an almost periodic group $T: J \rightarrow L(X, X)$. Further, suppose that $u: J \rightarrow X$, with its derivative $u^{\prime}(t) \in D(A)$ for ali $t \in J$, is a strong solution of the differential equation

$$
u^{\prime \prime}(t)=A u^{\prime}(t)+B(t) u(t)+f(t) \quad \text { a.e. on } \mathrm{J},
$$

where $B: J \rightarrow L(X, X)$ is almost periodic with respect to the norm of $L(X, X)$. If $u$ is $S^{1}$ - almost periodic and $u^{\prime}$ is $S^{1}$-bounded on $J$, then $u$ and $u^{\prime}$ are both almost periodic from $J$ to $X$.

2. LEMMAS.

LEMMA 1. The derivative of any solution of (1.9) has the representation

$$
u^{\prime}(t)=T(t) u^{\prime}(0)+\int_{0}^{t} T(t-s)[B(s) u(s)+f(s)] d s \text { on } J .
$$

PROOF. For an arbitrary but fixed $t \in J$, we have

$$
\begin{aligned}
\frac{d}{d s}[ & \left.T(t-s) u^{\prime}(s)\right]=T(t-s)\left[u^{\prime \prime}(s)-A u^{\prime}(s)\right] \\
& =T(t-s)[B(s) u(s)+f(s)] \text { a.e. on } J \text {, by (1.9). }
\end{aligned}
$$


Integrating (2.2) from 0 to $t$, we obtain the representation (2.1).

LEMMA 2. If $g: J \rightarrow X$ is an almost periodic function, and if $G: J \rightarrow L(X, X)$ is an almost periodic group, then $G(t) g(t), t \in J \rightarrow X$ is an almost periodic function ( $X$ a Banach space).

PROOF. See Zaidman [5].

LEMMA 3. Let $X$ be a reflexive Banach space, $h: J \rightarrow X$ an $S^{1}-$ almost periodic continuous function, and

$$
H(t)=\int_{0}^{t} h(s) d s \quad \text { on } J
$$

Then $H$ is almost periodic if it is $S^{1}$ - bounded on $J$.

PROOF. See Notes (ii) of Rao [4].

3. PROOF OF THEOREM.

From (2.1), we obtain

$$
T(-t) u^{\prime}(t)=u^{\prime}(0)+\int_{0}^{t} T(-s)[B(s) u(s)+f(s)] d s \text { on } J \text {. }
$$

We write

$$
v(t)=B(t) u(t)+f(t) \text { on } J
$$

Since $B$ is almost periodic from $J$ to $L(X, X)$, we have

$$
\sup _{t \in J}|B(t)|=M<\infty .
$$

Further, since $u$ is $S^{1}$-almost periodic from $J$ to $X$, it is $S^{1}$ - bounded on $J$.

Now, given $\epsilon>0$, we may choose $\tau$ to be an $\epsilon$ - almost period of $B$ and also an $e-S^{1}$ - almost period of $u$ (see pp. 10, 77 and 78, Amerio and Prouse [1]).

Then we have

$$
\begin{aligned}
\int_{t}^{t+1} & \mid B(s+\tau) u(s+\tau)-B(s) u(s) \| d s \\
& \quad \leq \int_{t}^{t+1}|B(s+\tau)-B(s)| \cdot\|u(s+\tau)\| d s \\
& +\int_{t}^{t+1}|B(s)| \cdot|u(s+\tau)-u(s)| d s \\
& \leq \in|u|_{S^{1}}+M e \text { on } J, \text { by }(1.2) \text { and }(3.3) .
\end{aligned}
$$

So $B(t) u(t)$ is $S^{1}$-almost periodic from $J$ to $X$. Hence $v$ is $S^{1}$ - almost periodic from $J$ to $X$.

Consider the function on $J$

$$
v_{h}(t)=\frac{1}{h} \int_{0}^{h} v(t+s) d s \text { for any } h>0
$$

Since $v$ is $S^{1}$-almost periodic, it follows easily that $v_{h}(t)$ is almost periodic for each fixed $h>0$. As shown for scalar-valued functions in Besicovitch [2], pp. 80-81, we can prove that $v_{h} \rightarrow v$ as $h \rightarrow 0+$ in the 
$S^{1}$ - sense, that is,

$$
\sup _{t \in J} \int_{t}^{t+1}\left\|v(s)-v_{h}(s)\right\| d s \rightarrow 0 \text { as } h \rightarrow 0+.
$$

Obviously, $T(-s), s \in J \rightarrow L(X, X)$ is an almost periodic group. So, for each $x \in X$, the function $T(-s) x$ is almost periodic, and hence is bounded on $J$. Thus, by the uniform boundedness principle,

$$
\sup _{s \in J}|T(-s)|=K<\infty .
$$

Now we have

$$
T(-s) v(s)=T(-s)\left[v(s)-V_{h}(s)\right]+T(-s) v_{h}(s),
$$

and, by $(3.7)$,

$$
\begin{aligned}
& \sup _{t \in J} \int_{t}^{t+1}\left\|T(-s)\left[v(s)-v_{h}(s)\right]\right\| d s \\
& \leq K \sup _{t \in J} \int_{t}^{t+1}\left\|v(s)-v_{h}(s)\right\| d s \rightarrow 0 \text { as } h \rightarrow 0+.
\end{aligned}
$$

By LEMMA 2, the functions $T(-t) v_{h}(t)$ are almost periodic from $J$ to $X$. Therefore it follows that $T(-t) v(t)$ is $S^{1}$ - almost periodic from $J$ to $X$.

Furthermore, by (3.7), $T(-t) u^{\prime}(t)$ is $S^{1}$ - bounded on $J$. Hence, by LEMMA $3, T(-t) u^{\prime}(t)$ is almost periodic from $J$ to $X$. Therefore, by LEMMA 2, $T(t)\left[T(-t) u^{\prime}(t)\right]=u^{\prime}(t)$ is almost periodic from $J$ to $X$. So $u^{\prime}$ is bounded on $J$, and hence $u$ is uniformly continuous on $J$. Consequently, by Theorem VII, p. 78, Amerio and Prouse [1], $u$ is almost periodic from $J$ to $X$, completing the proof of the theorem.

NOTE 2. In a reflexive space $X$, consider the first-order infinitesimal generator differential equations

$$
\begin{aligned}
& u^{\prime}(t)=[A+B(t)] u(t)+f(t) \quad \text { a.e. on } J, \\
& u^{\prime}(t)=A u(t)+f(t) \quad \text { a.e. on } J,
\end{aligned}
$$

where $f: J \rightarrow X$ is an $S^{1}$ - almost periodic continuous function, $A$ is the infinitesimal generator of an almost periodic group $T: J \rightarrow L(X, X)$, and $B: J \rightarrow L(X, X)$ is almost periodic with respect to the norm of $L(X, X)$. Then, from (3.10) and (3.11), we have the representations

$$
u(t)=T(t) u(0)+\int_{0}^{t} T(t-s)[B(s) u(s)+f(s)] d s \text { on } J
$$

and

$$
u(t)=T(t) u(0)+\int_{0}^{t} T(t-s) f(s) d s \text { on } J,
$$

respectively. From the proof of our THEOREM, it follows that, (a) if $u: J \rightarrow D(A)$ is an $S^{1}$-almost periodic solution of the differential equation (3.10), then it is almost periodic from $J$ to $X$, and (b) if $u: J \rightarrow D(A)$ is an $S^{1}$ - bounded solution of the differential equation (3.11), then it is almost periodic from $J$ to $X$. 


\section{REFERENCES}

1. AMERIO, L. and PROUSE, G. Almost Periodic Functions and Functional Equations, Van Nostrand Reinhold Company (1971).

2. BESICOVITCH, A.S. Almost Periodic Functions, Dover Publications Inc. (1954).

3. DUNFORD, N. and SCHWARTZ, J.T. Linear operators, Part I, Interscience Publishers Inc., New York (1958).

4. RAO, A.S. On Differential Operators with Bohr-Neugebauer Type Property, J. Diff. Eqs., 13 (1973), 490-494.

5. ZAIDMAN, S. Sur la Perturbation Presque-Périodique des Groupes et Semi-groupes de Transformations d'un Espace de Banach, Rend. Matem. e sue Appl.,16 (1957), 197-206. 


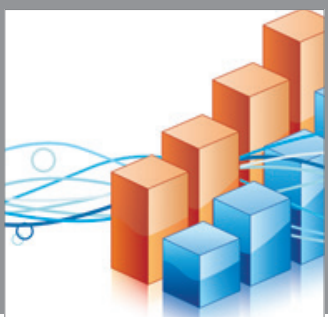

Advances in

Operations Research

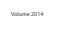

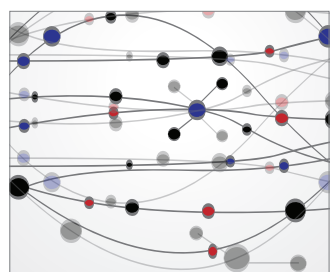

\section{The Scientific} World Journal
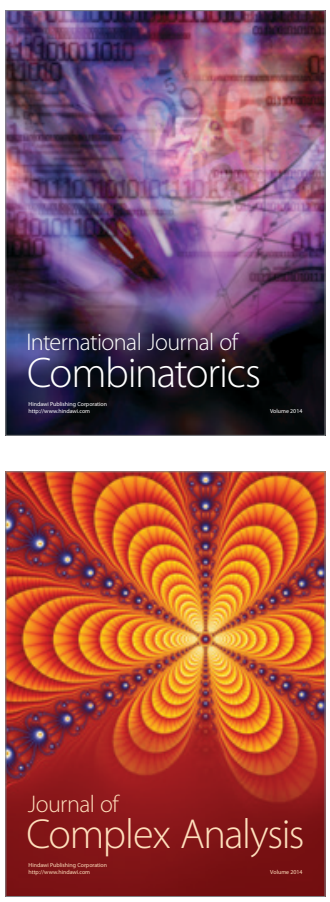

International Journal of

Mathematics and

Mathematical

Sciences
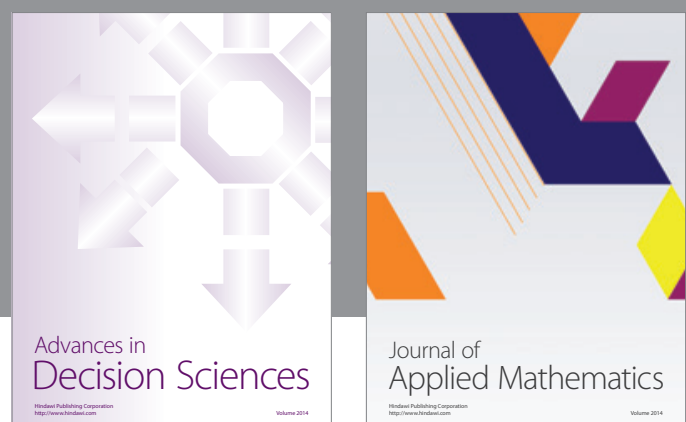

Journal of

Applied Mathematics
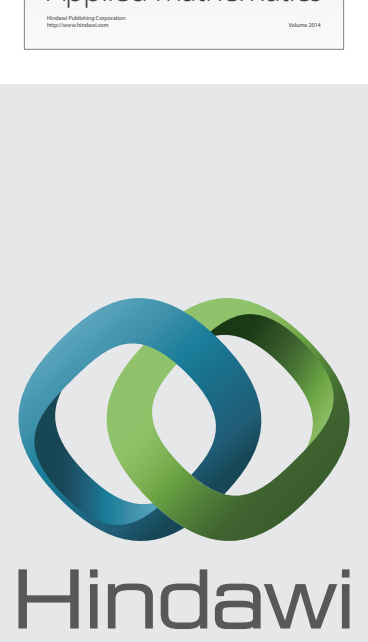

Submit your manuscripts at http://www.hindawi.com
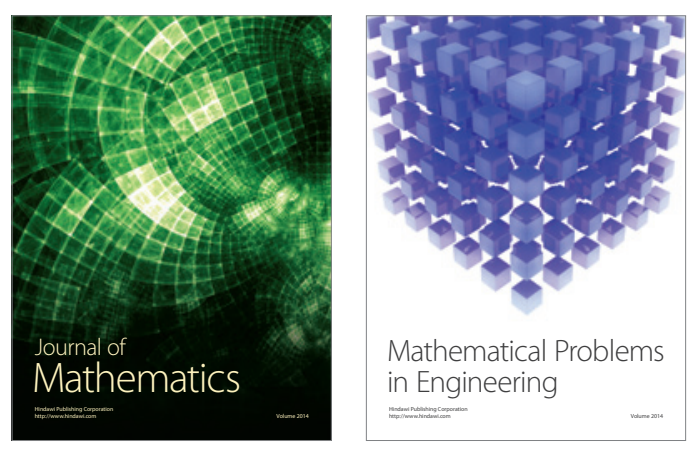

Mathematical Problems in Engineering
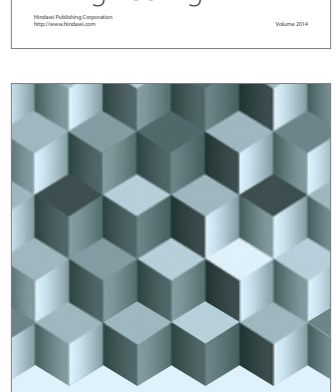

Journal of

Function Spaces
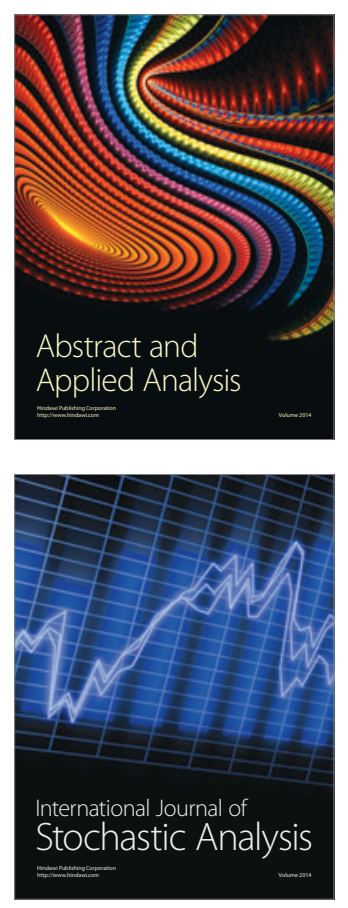

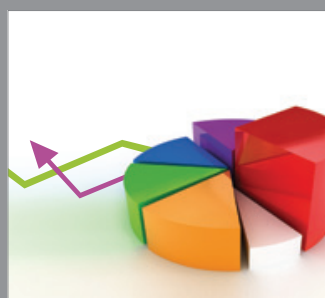

ournal of

Probability and Statistics

Promensencen
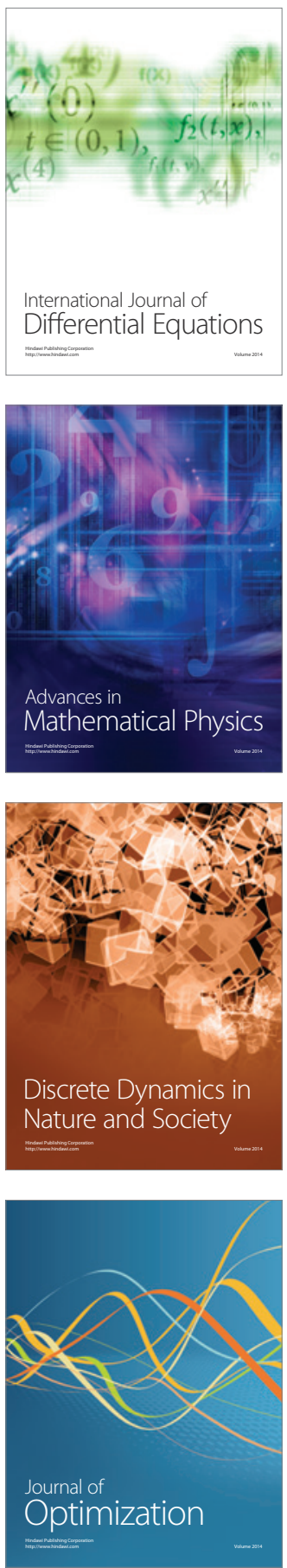